\title{
Measurements of diffractive structure functions with the LRG method and using the leading proton spectrometer at ZEUS
}

\author{
Jaroslaw Lukasik * \\ DESY/AGH-UST Cracow \\ Notkestrasse 85, D-22607 Hamburg - Germany
}

\begin{abstract}
The ZEUS detector has been used to study dissociation of virtual photons, $\gamma^{*} p \rightarrow X p$, in $e^{+} p$ collisions at HERA in events with a large rapidity gap (LRG) between $X$ and the outgoing proton, as well as in events with a measured leading proton. The data cover photon virtualities $Q^{2}>2 \mathrm{GeV}^{2}$, with $M_{X}>2 \mathrm{GeV}$, where $M_{X}$ is the mass of the hadronic final state $X$. The results are presented in terms of the diffractive structure functions, $F_{2}^{D(3)}$ and $F_{2}^{D(4)}$.
\end{abstract}

\section{Introduction}

The diagram of the diffractive Deep Inelastic ep Scattering (DIS) is shown in the Fig. 1. This process is characterised by the fact that $p$ loses a small fraction of its energy and emerges from the scattering intact or dissociated into a low-mass state with a transverse momentum squared typically much smaller than $1 \mathrm{GeV}^{2}$. The diffractive DIS events can be described, in addition to standard DIS variables, by the four-momentum transfer at $p$ vertex squared $t$ and invariant mass of $\gamma^{*} \mathbb{P}$ system $M_{X}$ which is the mass of the system resulting from virtual photon dissociation. If also proton dissociates into higher mass state it will be denoted by $N$. Moreover, the diffractive structure functions are often expressed in terms of $x_{\mathbb{P}}$ and $\beta$ variables. In a model with Pomeron exchange in the $t$ channel $x_{\mathbb{P}}$ is the fraction of the proton momentum carried by the Pomeron, while $\beta$ corresponds to

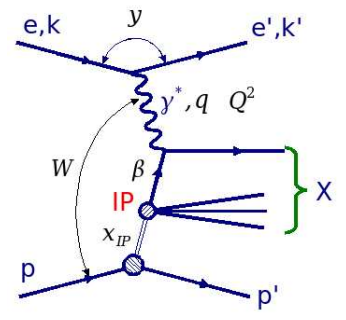

Figure 1: Diagram of the diffractive Deep Inelastic ep Scattering. the momentum fraction of the struck quark within the Pomeron.

The ZEUS collaboration used two different experimental approaches to select the inclusive diffractive events:

- measurement of the final state proton by means of a Leading Proton Spectrometer (LPS method) [2],

- a large rapidity gap in the forward direction requirement (LRG method).

The preliminary results obtained with the LPS and LRG methods will be presented in the following.

The data used for this measurement were taken with the ZEUS detector at HERA $e p$ collider in the year 2000, where HERA collided positrons of $27.6 \mathrm{GeV}$ with protons of 920 $\mathrm{GeV}$. The data used for the LRG and LPS analyses correspond to integrated luminosities of $45.4 \mathrm{pb}^{-1}$ and $32.6 \mathrm{pb}^{-1}$, respectively. The results presented here cover photon virtualities $Q^{2}>2 \mathrm{GeV}^{2}$, photon-proton centre-of-mass energies $40<W<240 \mathrm{GeV}$ and proton

${ }^{*}$ On behalf of the ZEUS Collaboration. 
fractional momentum losses $0.0002<x_{\mathbb{P}}<0.02$ (LRG sample) or $0.0002<x_{\mathbb{P}}<0.1$ (LPS sample).

\subsection{LPS method}

In most of the diffractive events outgoing proton stays intact and provides a clean experimental signature. Since $p_{t}$ of the outgoing proton is expected to be small (less than $1 \mathrm{GeV}$ typically), it escapes through the forward beam hole. A fraction of these events can be detected by the Leading Proton Spectrometer (LPS). In the spectrum of protons measured in the LPS shown as a function of $x_{L}=\left|p_{f}\right| /\left|p_{i}\right|$, where $p_{i}$ and $p_{f}$ are the initial and final proton momenta respectively [4], a characteristic peak is observed at $x_{L} \simeq 1$ which corresponds to photon diffractive dissociation events. A clean sample of diffractive events is obtained by requiring $x_{L}>0.97$. Measurement of the scattered proton four-momentum allows to study the $t$ distribution in inclusive diffractive dissociation.

\subsection{LRG method}

Experimental determination of the rapidity

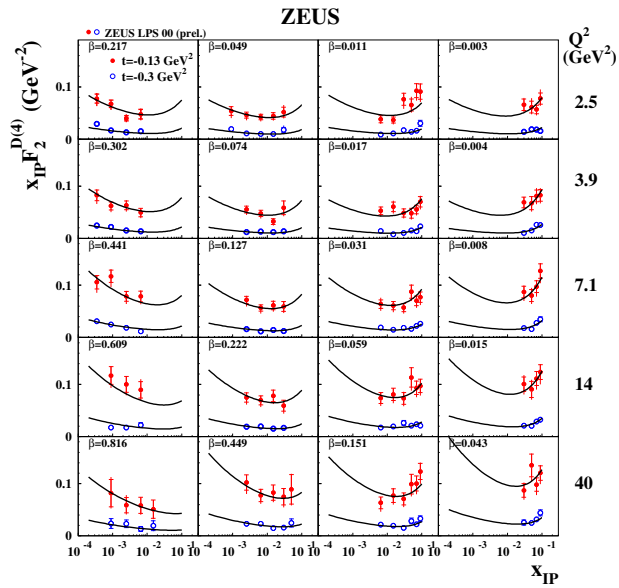

Figure 2: The diffractive structure function multiplied by $x_{\mathbb{P}}, x_{\mathbb{P}} F_{2}^{D(4)}$, in two $t$ bins as a function of $x_{\mathbb{P}}$ for different values of $Q^{2}$ and $\beta$. The normalisation uncertainty of $10 \%$ is not shown. The continuous lines are the result of the Regge fit described in the text.

gap rely on calculation of the pseudorapidity of the most forward going particle $\eta_{\max }$, which deposits some minimum amount of energy (above noise level) in the detector.

The $\eta_{\max }$ distribution in the DIS sample is characterised by a plateau like structure, due to diffractive events mainly, which extends to a low $\eta_{\max }$ values (large $\Delta \eta$ ). By setting an upper limit on the maximum pseudorapidity (e.g. $\eta_{\max }<3$ ), a diffractive sample with relatively low non-diffractive background can be selected.

\section{Results}

The data are presented in terms of the diffractive structure functions, $F_{2}^{D(3)}$ and $F_{2}^{D(4)}$. The results can be also presented in terms of the reduced cross section [3], $\sigma_{r}^{D(3)}\left(x_{\mathbb{P}}\right.$, which is equal to the conventional $F_{2}^{D(3)}$ up to corrections due to the longitudinal structure function.

The contributions from longitudinal structure function $F_{L}^{D}$ and $Z_{0}$ exchanges have been neglected in presented results. 


\subsection{Results from the LPS method}

Figure $2^{\mathrm{a}}$ shows the measured structure function $x_{\mathbb{P}} F_{2}^{D(4)}$. For $x_{\mathbb{P}}<0.01$ the structure function rises with decreasing $x_{\mathbb{P}}$. For higher values of $x_{\mathbb{P}}$ it starts to rise with increasing $x_{\mathbb{P}}$. This latter effect is attributed to the contributions from Regge trajectory exchanges reggeexch.

A sum of two contributions was fitted to the data according to

$$
F_{2}^{D(4)}=f_{\mathbb{P}}\left(x_{\mathbb{P}}, t\right) \cdot F_{2}^{\mathbb{P}}\left(\beta, Q^{2}\right)+n_{\mathbb{R}} \cdot f_{\mathbb{R}}\left(x_{\mathbb{P}}, t\right) \cdot F_{2}^{\mathbb{R}}\left(\beta, Q^{2}\right)
$$

The first term of this sum is the contribution from Pomeron exchange and the second one, from the exchanges of the Reggeon trajectories. The Reggeon structure function $F_{2}^{\mathbb{R}}\left(\beta, Q^{2}\right)$ was taken to be equal to the pion structure function as parametrised by GRV [5]. The fit was limited to $y<0.5$ to reduce the influence of $F_{L}^{D}$. Result of the fit is shown in the Fig. 2 .

\subsection{Results from the LRG method}

Inclusive diffractive data were selected with LRG method by requiring the maximum pseudorapidity to be $\eta_{\max }<3$ outside the Forward Plug Calorimeter (FPC) [7]. In addition events with energies in the FPC larger than $1 \mathrm{GeV}$ were rejected.

The resulting diffractive structure function $x_{\mathbb{P}} F_{2}^{D(3)}$ is shown in Figures 3-5. The result of a Regge fit, performed in the same way as described previously, is shown as the continuous lines. It gives a good description of the data. No rise of $x_{\mathbb{P}} F_{2}^{D(3)}$ coming from Reggeexchanges can be seen because the LRG data end essentially at $x_{\mathbb{P}}=0.01$.

The ratio of the $F_{2}^{D(3)}$ values obtained with the LPS method to the LRG data is shown in Fig. 6 up to $Q^{2}=40 \mathrm{GeV}^{2}$. The ratio is independent of $x_{\mathbb{P}}$ and equal in each $\beta$ and $Q^{2}$ bin with the average value $0.82 \pm 0.01$ (stat) \pm 0.03 (syst). Since up to $x_{\mathbb{P}}=0.01$ the LPS data contain no contribution from proton dissociation, this is an indication that the contribution from proton dissociation in the LRG data might be about $18 \%$. However, one has to take into account the large normalisation uncertainty of about $10 \%$ of the LPS data.

\section{Bibliography}

\section{References}

[1] Slides: http://indico.cern.ch/contributionDisplay $\cdot$ py?contribId=61\&sessionId=7\&conf Id=9499

[2] ZEUS Coll., M. Derrick et al., Z. Phys. C73 253 (1997).

[3] H1 Coll., A. Aktas et al., hep-ex/0606004.

[4] ZEUS Coll., J. Breitweg et al., Eur. Phys. J. C1 81 (1998)

[5] M. Glück, E. Reya and A. Vogt, Z. Phys. C53 127 (1992);

M. Glück, E. Reya and A. Vogt, Z. Phys. C53 651 (1992);

M. Glück, E. Reya and A. Vogt, Z. Phys. C67 433 (1995).

[6] P. D. B. Collins, An Introduction to Regge Theory and High Energy Physics, Cambridge University Press, Cambrige (1977).

[7] ZEUS Coll., FPC group, A. Bamberger et al., Nucl. Inst. Meth. A450 235 (2000).

\footnotetext{
${ }^{a}$ From now on in each plot the inner error bars will indicate the statistical uncertainties, the outer bars the statistical and systematic uncertainties summed in quadrature.
} 


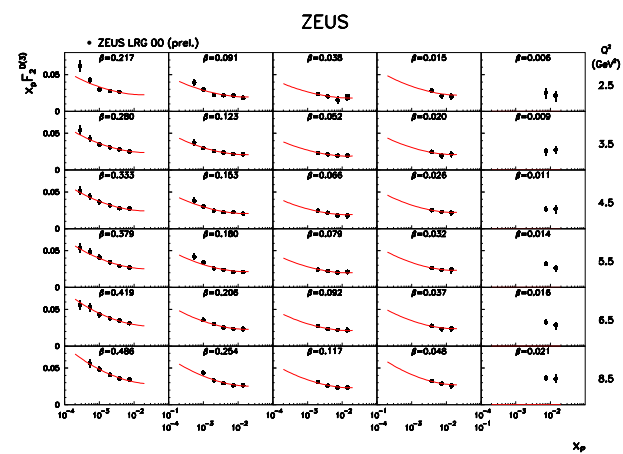

Figure 3: The diffractive structure function multiplied by $x_{\mathbb{P}}, x_{\mathbb{P}} F_{2}^{D(3)}$, obtained with the LRG method as a function of $x_{\mathbb{P}}$ for different values of $Q^{2}$ and $\beta$ at low $Q^{2}$ values. The normalisation uncertainty of $\pm 2.25 \%$ is not shown. The continuous lines are the result of the Regge fit described in the text.

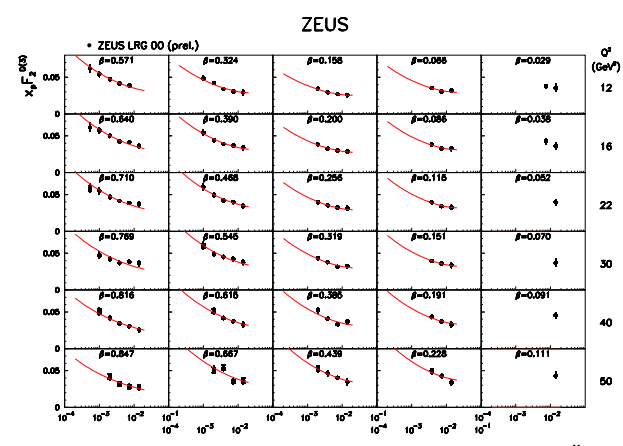

Figure 4: The diffractive structure function multiplied by $x_{\mathbb{P}}, x_{\mathbb{P}} F_{2}^{D(3)}$, obtained with the LRG method as a function of $x_{\mathbb{P}}$ for different values of $Q^{2}$ and $\beta$ at intermediate $Q^{2}$ values. The normalisation uncertainty of $\pm 2.25 \%$ is not shown. The continuous lines are the result of the Regge fit described in the text. 


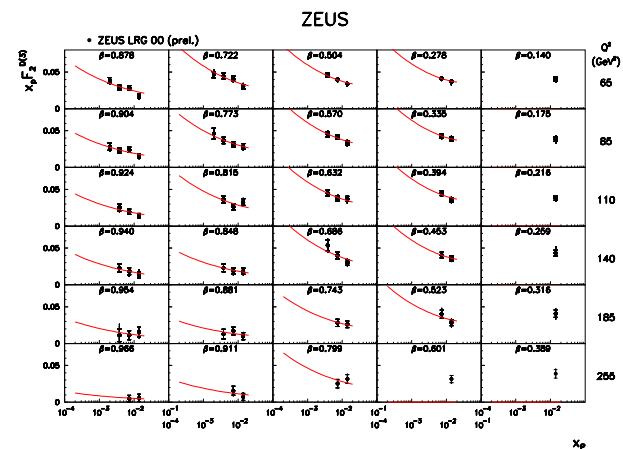

Figure 5: The diffractive structure function multiplied by $x_{\mathbb{P}}, x_{\mathbb{P}} F_{2}^{D(3)}$, obtained with the LRG method as a function of $x_{\mathbb{P}}$ for different values of $Q^{2}$ and $\beta$ at high $Q^{2}$ values. The normalisation uncertainty of $\pm 2.25 \%$ is not shown. The continuous lines are the result of the Regge fit described in the text.

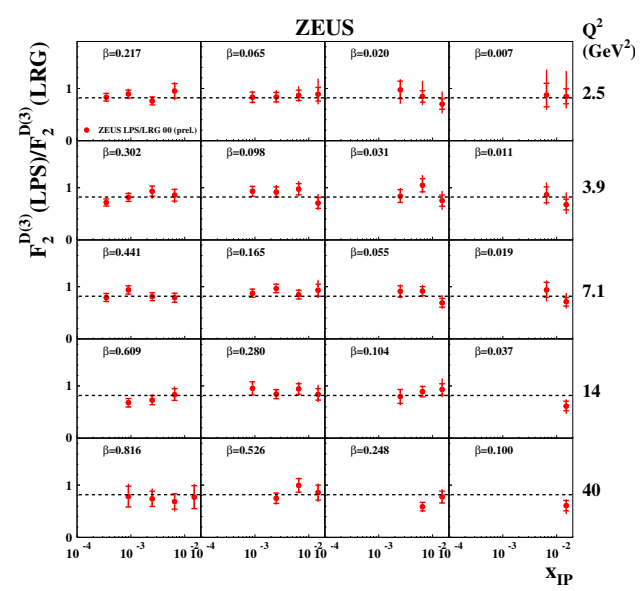

Figure 6: The ratio of the structure functions $F_{2}^{D(3)}$ as obtained with the LPS and LRG methods as a function of $x_{\mathbb{P}}$ for different values of $Q^{2}$ and $\beta$. The normalisation uncertainty of ${ }_{-10}^{+12} \%$ is not shown. The lines indicate the average value of the ratio. 